Bio - grafia. Escritos sobre la Biología y su Enseñanza. ISSN 2027

Edición Extraordinaria. p.p. 1590-1600

Memorias del VIII Encuentro Nacional de Experiencias en Enseñanza de la Biología y la Educación Ambiental. III Congreso Nacional de Investigación en Enseñanza de la Biología.

\title{
ANÁLISIS DE LAS CONCEPCIONES SOBRE EL CONCEPTO DE SISTEMA NERVIOSO EN ESTUDIANTES DE GRADO NOVENO (901) DEL COLEGIO ANTONIO NARIÑO I.E.D
}

\begin{abstract}
ANALYSIS OF CONCEPTIONS ON THE CONCEPT OF NERVOUS SYSTEM IN NINTH GRADE STUDENTS (901) OF ANTONIO NARIÑO I.E.D SCHOOL
\end{abstract}

Olave Chacón, Yenny Yadid'; Martín Urrego, Gina Esperanza²

\section{RESUMEN}

Las concepciones son ideas u opiniones que se adquieren en la vida cotidiana y el ambiente escolar a través de diferentes experiencias. La primera intervención didáctica evidencia las concepciones del concepto sistema nervioso, que poseen 6 estudiantes de grado noveno (901), con edades entre 14 y 17 años, de la ciudad de Bogotá D.C, del colegio Antonio Nariño de la jornada tarde. Las concepciones se analizaron bajo el paradigma cualitativo interpretativo; para el análisis se realizaron tres categorías emergentes; que se derivan a partir de los resultados obtenidos en el aula de clase: 1. Funcionalidad, 2. Estructuras Morfológicas y 3. Experiencias, basadas en la información obtenida del cuestionario que se aplicó. Se reconoció que los estudiantes aíslan el sistema nervioso de los demás sistemas, al mismo tiempo le atribuyen funciones motoras y sensitivas. Finalmente se evidencia en sus escritos que tienen errores conceptuales, y contradicen sus explicaciones.

PALABRAS CLAVES: concepción, paradigma cualitativo, sistema nervioso, categorías emergentes.

\section{ABSTRACT}

${ }^{1,2}$ Estudiante de Licenciatura en Biología, Integrantes del semillero de investigación Biología, Enseñanza y Realidades (BER), Integrantes del semillero y Grupo de Investigación en Ecología y Conservación de Plantas de Colombia (GIECPC). Universidad Distrital Francisco José de Caldas, Facultad de Ciencias y Educación. jennyyoch3@gmail.com; gina-martinurrego@hotmail.com 
Bio - grafia. Escritos sobre la Biología y su Enseñanza. ISSN 2027

Edición Extraordinaria. p.p. 1590-1600

Memorias del VIII Encuentro Nacional de Experiencias en Enseñanza de la Biología y la Educación Ambiental. III Congreso Nacional de Investigación en Enseñanza de la Biología.

The conceptions are ideas or opinions that acquired in life and school environment through different experiences. The next first teaching experience, shown conceptions of the concept nervous system, which have six freshmen of ninth grade (901), aged 14-17 years, in the city of Bogota DC, Antonio Nariño School. The conceptions were analyzed under the qualitative paradigm for the analysis three emerging categories were made; derived from the results obtained in the classroom: 1. Functionality, 2. and 3. Experiences morphological structures, based on information obtained from the questionnaire were applied. It was recognized that students insulate the nervous system of the other systems, while attributed motor and sensory functions and finally evidence in his writings that have conceptual errors, and contradict their explanations.

KEY WORDS: conception, qualitative paradigm, nervous system, emerging categories.

\section{INTRODUCCIÓN}

Esta intervención didáctica se basó en la realización de un análisis de las concepciones acerca del concepto del sistema nervioso de los estudiantes del grado noveno, con edades entre 14 y 17 años, de los cuales se toma un estudio de caso de 6 estudiantes del colegio Antonio Nariño I.E.D, jornada tarde de la ciudad de Bogotá D.C.

Comprender la complejidad del sistema nervioso, es posible mediante la elaboración de nuevos instrumentos, técnicas y conceptos que permiten comenzar a estudiar en detalle las estructuras nerviosas y su funcionamiento (Giordan, Raichvarg, Drouin, Gagliardi, \& Canay, 1988). Es importante comprender el concepto de sistema nervioso; ya que, incluso en la actualidad a pesar de los avances tecnológicos en medicina se observa como los estudiantes no se apropian de dicho concepto; que puede permitir el entendimiento del organismo y su ser. El sistema nervioso es único en la gran complejidad de reacciones de control que puede llevar a cabo. Puede recibir literalmente miles de datos de información procedentes de los diferentes órganos sensoriales y luego integrarlos todos para lograr la respuesta del cuerpo (Guyton, 1978). Es necesario implementar y crear nuevas formas de enseñanza del sistema nervioso para lograr el aprendizaje y la comprensión del concepto, dado que se observan dificultades de aprendizaje de este, porque se reduce y se vuelve mecanicista. 
Bio - grafia. Escritos sobre la Biología y su Enseñanza. ISSN 2027

Edición Extraordinaria. p.p. 1590-1600

Memorias del VIII Encuentro Nacional de Experiencias en Enseñanza de la Biología y la Educación Ambiental. III Congreso Nacional de Investigación en Enseñanza de la Biología.

Los alumnos poseen, previamente a las enseñanzas sistémicas sobre un objeto de estudio, un cierto número de ideas que denominamos "concepciones" (Giordan A. , 2000). Una concepción es el proceso de una actividad de construcción mental de lo real. Esta elaboración se efectúa a partir de las informaciones que se graban en la memoria y resultan de los sentidos y las relaciones con los otros (Giordan \& De Vecchi, 1995); las concepciones de los alumnos constituyen un referente fundamental para la construcción de un currículo escolar alternativo, socialmente más pertinente, que permita a la escuela participar efectivamente en la construcción de las sociedades del siglo XXI (García Díaz, 1998; García Pérez y De Alba, 2008 citado en González \& García-Pérez, 2014); las informaciones son codificadas, organizadas y categorizadas dentro de un sistema cognitivo global y coherente según las preocupaciones y los usos que de él hace cada cual. Giordan, (1995) afirma:

"La función de las concepciones es estructurar y organizar la realidad. Se ocupa de las situaciones en las que la persona debe resolver ciertos problemas, realizar diferentes actividades y concebir nuevas normas de conducta..." (p.110).

Según Osborne y Freyberg (1995) "si no sabemos lo que piensan los alumnos y por qué opinan así, tendremos escasas posibilidades de ejercer un impacto con nuestra enseñanza por muy hábil y adecuadamente que procedamos".

Así, pues el maestro investigador es quien tiene que analizar las diferentes concepciones de los estudiantes; puesto que a través del conocimiento de dichas, él podrá proponer una estrategia didáctica más eficaz en cuanto a sus componentes; situaciones, intervenciones del enseñante, ayudas didácticas y diseño didáctico (Giordan A. ,2000)

Autores como Silvia Bello (2004), Trinidad-Velasco \& Garritz (2003), Campanario \& Otero (2000) y Pozo (1996), han planteado que el reconocimiento y por lo tanto el análisis de las concepciones, idea previas o representaciones de los estudiantes acerca de un determinado concepto, contribuyen a que el docente pueda comprender el proceso de enseñanzaaprendizaje de las ciencias, para la futura elaboración de estrategias didácticas apropiadas en diferentes espacios. Para el análisis del sistema nervioso se encontraron trabajos de Gellert (1962) citado en Serrano (1988), Boujaada (1988) y Salazar (2013). 
Bio - grafia. Escritos sobre la Biología y su Enseñanza. ISSN 2027

Edición Extraordinaria. p.p. 1590-1600

Memorias del VIII Encuentro Nacional de Experiencias en Enseñanza de la Biología y la Educación Ambiental. III Congreso Nacional de Investigación en Enseñanza de la Biología.

Teniendo en cuenta estos referentes conceptuales el objetivo de esta primera intervención didáctica se centra en analizar las concepciones de los estudiantes sobre el sistema nervioso, conocer de dónde proceden esas concepciones y como ellos han llegado a lo declarativo. A través de categorías emergentes, estas se conciben desde el levantamiento de referenciales significativos a partir de la propia indagación (Cisterna, 2005).

\section{METODOLOGÍA}

Nieto \& Rodríguez (2009), plantean que el paradigma cualitativo interpretativo radica en las nociones tales como comprensión, significado y acción; pone su acento en el mundo personal de los sujetos, interpretando las situaciones, el significado, las intenciones propias de los sujetos, buscando como criterio de objetividad los significados intersubjetivos evidentes que se puedan producir en el contexto educativo.

Para recoger la información acerca de las ideas previas de los estudiantes del grado noveno (901) de la jornada tarde del colegio Antonio Nariño I.E.D, se realizó y se aplicó un instrumento basado en los postulados de Cubero (1989) en el texto ¿Cómo trabajar con las ideas de los alumnos?; se realizó un cuestionario con 2 preguntas, ¿Qué sé acerca del sistema nervioso? Y ¿Qué me gustaría saber del sistema nervioso?; de las cuales se tomó la primera pregunta para el análisis, que permitió explorar lo que conocen los estudiantes acerca de dicho tema. De estos instrumentos se tomaron como estudio de caso seis estudiantes de los treinta pertenecientes al grado noveno; ya que fueron los que recogían los pensamientos de la mayoría de estudiantes y algunas concepciones únicas. Estos cuestionarios se transcribieron tal cual estaban escritos. A través de esto se va a tratar de comprender como los estudiantes llegaron a crear esas concepciones en el aula de clase. Se utilizaron tres categorías emergentes que se derivaron de los resultados para su posterior análisis; Funcionalidad: en la cual el estudiante describe los procesos que realiza el sistema nervioso, Estructuras morfológicas: en esta categoría el estudiante expresa como se encuentra estructurado el sistema nervioso a nivel de órganos y finalmente tenemos las Experiencias: donde los estudiantes narran vivencias o hechos de su vida fuera del aula de clases.

\section{RESULTADOS Y ANÁLISIS}


Bio - grafia. Escritos sobre la Biología y su Enseñanza. ISSN 2027

Edición Extraordinaria. p.p. 1590-1600

Memorias del VIII Encuentro Nacional de Experiencias en Enseñanza de la Biología y la Educación Ambiental. III Congreso Nacional de Investigación en Enseñanza de la Biología.

Se exponen los siguientes resultados para la actividad en la cual los estudiantes plantearon de forma escrita lo que saben acerca del sistema nervioso, bajo tres categorías, para clasificar las expresiones de cada estudiante, señalando entre paréntesis el número del cuestionario del cual fue tomada tal expresión (Cuestionario $1=C 1$, Cuestionario $2=C 2$, Cuestionario $3=C 3$, Cuestionario $4=C 4$, Cuestionario $5=C 5$, Cuestionario $6=C 6$ ) para el posterior análisis.

Tabla 1. Categorización de las respuestas de los estudiantes de grado noveno (901) a la pregunta ¿Qué sé acerca del sistema nervioso?

\begin{tabular}{|l|l|}
\hline Categoría & Expresiones \\
\hline Funcionalidad & "Sistema de estímulos que recorre nuestro cuerpo, nos da la impresión \\
del dolor igual que otro tipo de estímulos" (C1), (C3). \\
"Sé que el sistema nervioso controla las acciones de su o nuestro cuerpo \\
(C2). \\
"Es el que rige todos los movimientos del cuerpo y los músculos" (C3). \\
"Sistema nervioso central: es por donde corre todos nuestros \\
movimientos y sensaciones. \\
Sistema nervioso periférico: es los movimientos que hacemos \\
intencionalmente como el hipo y el latido del corazón etc. \\
Sistema nervioso autónomo: lo que podemos controlar como el \\
estornudo, el parpadeo etc." (C6). \\
"El sistema nervioso está compuesto y constituido por todos los \\
estímulos..." (C5).
\end{tabular}


Bio - grafia. Escritos sobre la Biología y su Enseñanza. ISSN 2027

Edición Extraordinaria. p.p. 1590-1600

Memorias del VIII Encuentro Nacional de Experiencias en Enseñanza de la Biología y la Educación Ambiental. III Congreso Nacional de Investigación en Enseñanza de la Biología.

\begin{tabular}{|l|l|}
\hline "El sistema nervioso es cuando nos pican con un palo o las hormigas, \\
sentimos alguna cosa, como sorprendido. De eso se trata el sistema \\
nervioso, de sentir lo que nos ha tocado picado" (C4). \\
"...y cosas y que sentimos frente a cualquier reacción y reflejos que \\
podamos o nos pueden ocurrir en el transcurso de nuestros días. Dichos \\
estímulos tales como la risa y todos y cada uno de los sentidos" (C5).
\end{tabular}

\section{Categoría 1. Funcionalidad.}

Los estudiantes usan términos como reacción, estímulos, coordinación, que son funciones propias del sistema nervioso, pero no los explican; por ejemplo no se sustenta cómo se da el proceso cuando el entorno manda información que es recibida a través de los órganos de los sentidos, a la que se da respuesta, normalmente, mediante un acto voluntario, que es controlado por la corteza cerebral, otras veces, la respuesta se da mediante actos reflejos que son respuestas rápidas y cortas, controladas por la médula espinal, sin que intervenga el encéfalo. No hay comprensión de los términos que usan, en sus explicaciones, apropian expresiones de sentido común (Salazar, 2013).

A través de la función, los estudiantes intentan explican el concepto por su utilidad, reduciéndolo así a una de sus cualidades. La generalización del concepto puede llegar a crear equivocaciones, ya que deja de lado los detalles que permiten que el concepto tenga sentido y validez científica (Salazar, 2013).

Los estudiantes no tienen claridad acerca de la diferencia entre lo que es la función y la estructura de sistema nervioso, por lo tanto dan expresiones tales como: "El sistema nervioso está compuesto y constituido por todos los estímulos" C5; se puede evidenciar que ellos en su afán de contestar al cuestionario no hacen un análisis minucioso de lo que se pregunta. A su vez, ellos poseen una confusión por la clasificación que tiene científicamente el sistema nervioso, donde se clasifica anatómicamente y funcionalmente (Guyton, 1978). Se deduce que ellos confunden estos modelos, posiblemente por las explicaciones que han dado los docentes en cursos anteriores donde hay un tiempo delimitado para la enseñanza de este sistema, donde lo importante no es la calidad del contenido enseñado si no la cantidad de contenidos que se enseñen. A su vez, se observa en sus escritos que tienen errores conceptuales y contradicen sus explicaciones, se evidencia en expresiones tales 
Bio - grafia. Escritos sobre la Biología y su Enseñanza. ISSN 2027

Edición Extraordinaria. p.p. $1590-1600$

Memorias del VIII Encuentro Nacional de Experiencias en Enseñanza de la Biología y la Educación Ambiental. III Congreso Nacional de Investigación en Enseñanza de la Biología.

como: "Sistema nervioso periférico: es los movimientos que hacemos intencionalmente como el hipo y el latido del corazón etc." C6. Esta expresión no da cuenta de que existe el sistema nervioso somático que tiene funciones voluntarios. Además, dicen: "Sistema nervioso autónomo: lo que podemos controlar como el estornudo, el parpadeo etc." C6, aquí se demuestra que los estudiantes tienen un error conceptual con la definición que le atribuyen a la palabra autónomo, esto significa que se "gobierna por sí mismo" (DRAE, 2014), lo cual no quiere decir que sea el individuo en sí, sino más bien el sistema del cual se compone. Otro aspecto a analizar son las funciones biológicas que tienen los individuos, voluntarias e involuntarias, los estudiantes no las reconocen como tal por lo que dicen que el hipo y los latidos del corazón son intencionales, es decir, que ellos pueden elegir su realización (Guyton, 1978).

Categoría 2. Estructuras morfológicas.

Según Jhonson y Wellman (1982) citado en Serrano (1993), las ideas de los niños muestran una tendencia creciente a asociar el cerebro con actos motores y sensitivos; los estudiantes reconocen que el cerebro y los nervios son una parte morfológica del sistema nervioso, pero no integran los demás órganos que lo constituyen, tales como: medula espinal, cerebelo, etc.; por lo tanto ven el cerebro como un todo y al mismo tiempo lo independizan de cada uno de los sistemas que compone el cuerpo humano; se infiere que piensan así porque es un órgano muy grande y abarca casi todo la cavidad craneal. En el contexto donde se desarrollan los individuos se usan expresiones como "usen su cerebro para todo", es aquí donde los estudiantes empiezan a formar concepciones. Gellert (1962) citado en Serrano (1993), explica que los niños entre la edad entre 13 y 17 años, empiezan a tener concepciones de los nervios como conductores de mensajes entre el cerebro y diferentes partes del cuerpo, o como elementos de aviso y protección.

\section{Categoría 3. Experiencias.}

Los estudiantes pueden tener experiencias previas o vivencias propias o cercanas en familiares o amigos que tienen algún grado de consumo de alguna sustancia psicoactiva, ya que relacionan el sistema nervioso con la pérdida de funciones, tales como: el lenguaje, el comportamiento, la motricidad por el uso de esas sustancias. Asimismo, hacen alusión a la inhibición de neurotransmisores por el consumo de esas sustancias, los estudiantes tienen claro que las drogas son nocivas para la salud, sin embargo no saben cómo actúan en el organismo, ni que el daño que provocan no es visible físicamente, pero si a nivel neuronal, ya que el exceso de estas sustancias que actúan como antagonistas de los neurotransmisores, 
Bio - grafia. Escritos sobre la Biología y su Enseñanza. ISSN 2027

Edición Extraordinaria. p.p. 1590-1600

Memorias del VIII Encuentro Nacional de Experiencias en Enseñanza de la Biología y la Educación Ambiental. III Congreso Nacional de Investigación en Enseñanza de la Biología.

afectan la transmisión del impulso nervioso y producen un cambio en la bioquímica del cerebro (Salazar, 2013).

Ellos reconocen algo sumamente importante y es que el sistema nervioso es "delicado" C2, se considera que la convivencia con sus padres le aportaron conocimientos cotidianos sobre el cuidado de su cuerpo, principalmente el cuidado de su cabeza. Al mismo tiempo, el estudiante no reconoce las diferentes estructuras morfológicas que pueden verse afectadas como lo son: el cerebro, la medula, el cerebelo, el bulbo raquídeo, los nervios entre otros.

El aprendizaje y los aprendizajes concretos dependen de estas ideas. El estudiante interpreta las informaciones a través de las concepciones que posee (Giordan A. , 2000). Así pues, se puede inferir que los estudiantes asocian sus experiencias que pueden percibir a través de los sentidos, en diferentes ámbitos de su vida cotidiana, principalmente asocian el sistema nervioso con el dolor, ya que por diferentes cuestiones han tenido un acercamiento con el sentido del tacto.

Los niños como científicos desarrollan ideas, aunque sea de forma tácita, acerca de cómo y por qué las cosas son como son; ideas que a cada uno en particular le parecen coherentes; son justamente esas ideas que nosotros denominamos "la ciencia de los alumnos" (Osborne, 1980; Giber, Osborne y Fensham, 1982 citado en Osborne y Freyberg 1995). Por lo cual, los estudiantes siempre llegaran al aula de clase con un conocimiento bien sea correcto 0 incorrecto en el ámbito científico, y dicho conocimiento tiene unas nociones y un fundamento en la vida del sujeto. Así, los docentes deben interpretar porque el estudiante imagina otro mundo y conocerlo es muy importante, haciendo una relación estrecha entre el pensamiento y el lenguaje.

A pesar de contar con este instrumento de caracterización de las ideas previas de los estudiantes, no podemos generalizar y predecir que dichas sean la totalidad de las concepciones que manejan los estudiantes, porque la actividad de recogida de información se da en un espacio convencional como lo es un aula de clase y quizás muchos estudiantes pudieron escribir las ideas solo por contestar a la ligera. Según Giordan (1995), las representaciones no son evidentes y no se pueden descubrir de inmediato, ya que las 
Bio - grafia. Escritos sobre la Biología y su Enseñanza. ISSN 2027

Edición Extraordinaria. p.p. 1590-1600

Memorias del VIII Encuentro Nacional de Experiencias en Enseñanza de la Biología y la Educación Ambiental. III Congreso Nacional de Investigación en Enseñanza de la Biología.

"concepciones", dice él, son modelos explicativos subyacentes. Se intentó inferir el por qué los estudiantes llegaron a esas concepciones, pero es necesario obtener más información de los estudiantes para comprender en su totalidad de donde provienen esas concepciones.

CONCLUSIONES

- A partir del análisis y la caracterización que se realizó de las concepciones de los estudiantes se evidenció las falencias que tienen al definir el concepto de sistema nervioso por las respuestas dadas. Por lo tanto se observó que los estudiantes hacen alusión a explicar el sistema nervioso a partir de las experiencias de su vida cotidiana.

- Los estudiantes no tienen claridad acerca de la diferencia entre lo que es la función y la estructura de sistema nervioso, por lo tanto, ellos tienden a definir el concepto por su utilidad y lo comprimen solo a una de sus cualidades. Igualmente los estudiantes tienden a desligar los órganos que lo integran, dejando el cerebro como un todo.

- Las categorías emergentes permitieron clasificar las concepciones de los estudiantes, para luego realizar un análisis más detallado de sus expresiones ante la pregunta propuesta en el instrumento de ideas previas.

- La primera intervención didáctica en el aula de clase es relevante en el proceso de formación de los docentes noveles; puesto que, se fomentan los procesos de investigación en el desarrollo profesional de dichos.

\section{Referencias}

Bello, S. (Julio de 2004). Ideas previas y cambio conceptual. Educación química., 15(3), 210217.

Boujaada, E. (1988). s'ehiculéesparle díscours dw, manuel de óiologie maocain au secotadaire, et leur incidence sur la conception de lapprensissage. Paris: Universite Laval.

Campanario, J. M., \& Otero, J. C. (2000). Más allá de las ideas previas como dificultades de aprendizaje. Enseñanza de las ciencias, 18, 155-159.

Cisterna, F. (2005). Categorización y triangulación como procesos de validación del conocimiento en investigación cualitativa. Theoria, 14(1), 61-71. 
Bio - grafia. Escritos sobre la Biología y su Enseñanza. ISSN 2027

Edición Extraordinaria. p.p. 1590-1600

Memorias del VIII Encuentro Nacional de Experiencias en Enseñanza de la Biología y la Educación Ambiental. III Congreso Nacional de Investigación en Enseñanza de la Biología.

Cubero, R. (1989). Cómo trabajar con las ideas de los alumnos. Dialnet.

DRAE. (Octubre de 2014). Real Academia Española. (e. 23. ${ }^{a}$, Editor) Obtenido de DRAE: http://www.rae.es/recursos/diccionarios/drae

Giordan, A. (2000). La enseñanza de las ciencias. Planteamientos en educación. Santafé de Bogotá, Colombia: Escuela pedagogica experimental.

Giordan, A., \& De Vecchi, G. (1995). Los orígenes del saber: de las concepciones personales a los conceptos científicos. Investigación y aprendizaje. Sevilla, España: Díada Editora S.I.

Giordan, A., Raichvarg, D., Drouin, J. M., Gagliardi, R., \& Canay, A. M. (1988). Conceptos de Biología. 1 La respiracion. Los microbios. El ecosistema. La neurona. Madrid: Labor S.A.

González Puentes, F., \& García-Pérez, F. F. (2014). Las concepciones de los alumnos sobre el medio urbano y sus implicaciones didácticas. Un estudio en Bogotá. Didáctica Geográfica, 41-60.

Guyton, A. (1978). Anatonmía y fisiología del sistema nervioso. México: Interamericana.

Osborne, R., \& Freyberg, P. (1995). El aprendizaje de las ciencias. Implicaciones de las "ideas previas" de los alumnos. Madrid: Narcea, S.A. de ediciones.

Pozo, J. I. (1996). "Las ideas del alumnado sobre la ciencia: de dónde vienen, a dónde van... y mientras tanto qué hacemos con ellas." Alambique: Didáctica de las ciencias experimentales 3.7 (1996): 18-26. Alambique: Didáctica de las ciencias experimentales, 3(7), 18-26.

Salazar, L. (2013). Enseñanza aprendizaje del concepto de sistema nervioso en estudiantes de básica secundaria.

Serrano, T. (1993). Desarrolo conceptual del sistema nervioso en niños de 5 a 14 años. Modelos mentales A-C. Madrid: Universidad Complutense de Madrid, Servicio de Publicaciones. 
Bio - grafia. Escritos sobre la Biología y su Enseñanza. ISSN 2027

Edición Extraordinaria. p.p. 1590-1600

Memorias del VIII Encuentro Nacional de Experiencias en Enseñanza de la Biología y la Educación Ambiental. III Congreso Nacional de Investigación en Enseñanza de la Biología.

Trinidad-Velasco, R., \& Garritz, A. (2003). Revisión de las concepciones alternativas de los estudiantes de secundaria sobre la estructura de la materia. Educación química. Educación química, 14(2), 92-105. 